\title{
Atrição dental em Didelphis albiventris e D. marsupialis (Marsupialia, Didelphimorphia, Didelphidae) do Sul do Brasil
}

\author{
Dental wear in Didelphis albiventris and D. marsupialis (Marsupialia, \\ Didelphimorphia, Didelphidae) from southern Brazil
}

\author{
Marília da Silva Aguiar ${ }^{1}$ Jorge Ferigolo $^{2}$ João Luiz Rossi Junior ${ }^{3}$ \\ Marco Antônio Gioso 4
}

\section{RESUMO}

Pesquisou-se o nível de desgaste dental (atrição el ou abrasão) em 168 espécimes de Didelphis albiventris e D. marsupialis (gambás) do sul do Brasil. O desgaste foi muito importante, com elevada freqüência de dentina terciária e de exposição da câmara coronária elou do canal radicular, devido à associação de fraturas e desgaste, pois as fraturas predispõem ao desgaste e este favorece as fraturas. $O$ grau de desgaste aumentou com a idade dos animais. A ordem crescente na freqüência de dentina terciária nos dentes caudais aos caninos foi: primeiro, segundo e terceiro molares, terceiro e segundo pré-molar, e quarto molar. Diferentemente de outros onívoros, em Didelphis o desgaste dentário parece mais relacionado às fraturas devidas aos alimentos (seguida de atriçãolabrasão) do que ao contato entre os dentes. Ao contrário da literatura, os molares tribosfênicos não parecem "ideais" para tal dieta onívora, o que é evidenciado pela elevada freqüência de desgaste, fraturas e exposição da câmara coronária ou do canal radicular.

Palavras-chave: Didelphis, desgaste, atrição, abrasão, dentina terciária, fraturas.

\section{ABSTRACT}

This work deals with the study of dental wear down (atrittion and/or abrasion) in 168 specimens of Didelphis albiventris and D. marsupialis from Southern Brazil. Wear down was very important, with high frequency of tertiary dentine and exposition of the pulp cavity/radicular canal, due to the association of fractures and wear down. Fractures predispose wear down which, on the other side, favors fractures. The degree of attrition increased with age. The order of growing frequency of tertiary dentin in poscanines was: first, second and third molars, third and second premolars, and finally fourth molar. Differently from others omnivorous in Didelphis tooth wear down seems rather related to fractures due to food (followed by attrition) than to teeth contact. Contrary to literature, tribosphenic molars seems not to be "ideals" for such omnivorous diet, as is evident from the high frequency of wear down, fractures and teeth cavities exposition.

Key words: Didelphis, wear, attrition, abrasion, tertiary dentin, fractures.

\section{INTRODUÇÃO}

Pesquisas recentes vêm relacionando continuamente a saúde oral com a sistêmica, tornando a odontologia veterinária cada vez mais importante (GIOSO, 1997). No entanto, tal desenvolvimento foi tardio na clínica médica veterinária preventiva de animais selvagens, pois as lesões da cavidade oral nestes animais não costumam ser eficientemente compreendidas e documentadas pelos profissionais que trabalham com tais espécies (KAZIMIROFF, 1938; ROBINSON, 1979).

Os animais do gênero Didelphis são onívoros e muito pouco seletivos em relação à sua dieta, que é composta principalmente de insetos, aves, ovos, pequenos mamíferos, frutas, sementes, folhas e, ocasionalmente, répteis, anfíbios e moluscos, dependendo da época do ano e da disponibilidade de alimentos. A di-

${ }^{1}$ Pesquisador, MSc, AGRODEFESA, Centro de Diagnóstico e Pesquisas Veterinárias, Rodovia R2, s/n, Campus II, Saída para Nova Veneza, 74670-310, Goiânia-GO. E-mail: mariliaaguiar@zipmail.com.br. Autor para correspondência.

${ }^{2}$ Pesquisador, Doutor, Museu de Ciências Naturais, Fundação Zoobotânica do Rio Grande do Sul (FZB/RS).

${ }^{3}$ Médico Veterinário, Mestrando, Departamento de Cirurgia, Faculdade de Medicina Veterinária e Zootecnia (FMVZ), Universidade de São Paulo (USP).

${ }^{4}$ Professor, PhD, FMVZ-USP. 
eta dos animais mais jovens consiste principalmente de invertebrados, frutas e plantas, enquanto os indivíduos com mais idade alimentam-se também de pequenos vertebrados (CORDERO \& NICOLAS, 1987; SANTORI et al., 1995).

Nos mamíferos carnívoros e onívoros (a maioria braquiodonte), o desgaste por atrição é relativamente menor do que nos herbívoros (DUBIELZIG, 1990), devido ao fato da dieta ser menos abrasiva e ter um conteúdo energético maior e, portanto, ser necessária em menor quantidade. Apesar de Didelphis ser onívoro, apresenta desgaste muito intenso, até mesmo em espécimes relativamente jovens (FERIGOLO, 1995), apesar da ausência de elementos abrasivos na sua dieta (como nos herbívoros).

Atrição é o desgaste fisiológico das estruturas dentais em decorrência da mastigação e está associada ao processo normal de envelhecimento, enquanto abrasão é o desgaste patológico e ocorre devido a processo mecânico anormal (SHAFER et al., 1979) como, por exemplo, o hábito de certos animais em cativeiro de friccionar os dentes nas barras de ferro do recinto. Desta forma, desgaste refere-se tanto à atrição quanto à abrasão.

Em Didelphis, além da atrição, ocorre perda de estruturas dentais em função da abrasão, que ocorre quando o animal "fricciona" os caninos superiores contra os inferiores na intenção de produzir som durante o comportamento de corte, encontros agressivos entre os adultos e quando a mãe quer chamar os filhotes (GARDNER, 1982), contribuindo para a formação de dentina terciária no terço oclusal dos caninos.

Os incisivos, segundo HIIEM $\ddot{A E} \&$ CROMPTON (1971), somente servem para prender os alimentos (sendo, portanto, "órgãos de apreensão") os quais são imediatamente transferidos para a região distal aos caninos. De acordo com CROMPTON \& HIIEMÄE (1970), no começo da mastigação o volume do alimento é grande, sendo ele então perfurado e triturado pelas altas e pontiagudas cúspides dos molares e pré-molares, sem que haja contato oclusal. Isto corresponde a mais de $60 \%$ do tempo gasto na mastigação, e produz rápido desgaste por atrição nas pontas das cúspides. Depois de um longo período de trituração, o alimento é, então, cortado pelos declives das cúspides dos molares superiores e inferiores, que agem como se fossem tesouras, através do movimento ântero-medial da pré-protocristida contra a metacrista, que são as maiores e mais desenvolvidas superfícies de corte.

Toda mastigação em $\boldsymbol{D}$. marsupialis é cíclica, rítmica e repetitiva, e ocorre em um lado por mais de 30 vezes, dependendo do tipo de alimento, antes que os lados sejam trocados (HIIEMÄE \& CROMPTON, 1971).

Ainda de acordo com HIIEMÄE \& CROMPTON (1971), “... as múltiplas faces de corte e as cúspides pontiagudas do molar tribosfênico em Didelphis são ideais para uma dieta de insetos, pequenos animais, plantas macias e frutas". Apesar disto, Didelphis pertence a um táxon que apresenta uma das mais elevadas freqüências de lesões decorrentes de grande desgaste dental, que se torna mais evidente nos animais mais velhos: "em Didelphis jovem, as maiores cúspides apresentam algum desgaste e, à medida que envelhecem, as cúspides são rapidamente desgastadas e reduzidas a superfícies lisas" (CROMPTON \& HIIEMÄE, 1970). Além disso, a perda de um dente ou da sua coroa, devido a desgaste ou fratura, traz como resultado a aplicação de toda força mastigatória sobre um reduzido complexo de dentes, aumentando o ritmo de perda de estruturas dentais (SPOUGE, 1977).

Segundo FERIGOLO (1995), o fato de o único dente substituído ser o terceiro pré-molar primário explicaria o grande desgaste encontrada em Didelphis, principalmente nos indivíduos com mais idade. Acredita-se que a este fator estejam aliados outros, como o importante papel que a mastigação potente, com músculos extremamente desenvolvidos (HIIEMÄE \& CROMPTON, 1971), tem no desgaste por atrição encontrado em Didelphis. FERIGOLO (1995) sugere que num processo tão grande de desgaste, como o encontrado em Didelphis, a perda da funcionalidade, além da dor pronunciada possam ser os principais fatores limitantes do tempo de vida destes animais, pois a dificuldade em mastigar pode fazer com que se alimentem cada vez menos.

\section{MATERIAL E MÉTODOS}

Foram estudados 168 sincrânios (crânio e mandíbula) de Didelphis albiventris e D. marsupialis pertencentes aos acervos da Coleção de Mastozoologia do Museu de Ciências Naturais da Fundação Zoobotânica do Rio Grande do Sul (MCN-M), da Coleção de Mamíferos Terrestres da Universidade Federal de Santa Catarina (UFSC), bem como do Museu de História Natural Capão da Imbuia (MHN-CI), Paraná. A maior parte do material pertencente à coleção do MHN-CI não havia sido tombada quando da realização deste estudo, razão pela qual apresentavam a sigla (CTX) e numeração provisória.

O estudo foi desenvolvido no Museu de Ciências Naturais da Fundação Zoobotânica do Rio 
Grande do Sul, Porto Alegre. A observação foi macroscópica, utilizando-se também um esteromicroscópio Zeiss para melhor visualização do nível de desgaste dental.

Os dentes foram referidos pelas seguintes siglas: I/i- incisivo, $\mathrm{C} / \mathrm{c}$ - canino, $\mathrm{P} / \mathrm{p}$ - pré-molar, e $\mathrm{M} /$ $\mathrm{m}$ - molar, respectivamente superiores/inferiores. A fórmula dental utilizada neste trabalho foi a de PAULACOUTO (1979): 5.1.3.4/4.1.3.4, isto é: cinco incisivos, um canino, três pré-molares e quatro molares no hemi-arco superior; para o hemi-arco inferior, são quatro incisivos, um canino, três pré-molares e quatro molares. Considerou-se que o único dente que é substituído é o terceiro pré-molar decíduo (ou primário) pelo terceiro pré-molar permanente (ou secundário). No intuito de facilitar a localização das lesões coronárias, adotou-se a terminologia anatômica aplicada à odontologia humana.

A fim de estabelecer a idade dos indivíduos a que pertenciam os sincrânios com base no desgaste dental e na deposição de dentina foi utilizada uma escala de classificação dental pelo grau de desgaste segundo DAVIES \& PEDERSEN (1955), porém adaptada, pois quando se refere à dentina secundária, no nível 2 , trata-se na verdade de dentina terciária, visto ser uma formação reacional à atrição de estrutura mineralizada adjacente aos odontoblastos, o que é análogo a outras espécies (WIGGS \& LOBPRISE, 1997; GIOSO, 2002): Nível 0 - sem desgaste; Nível 1 - desgaste somente no esmalte; Nível 2 - formação de dentina terciária; Nível 3 - exposição da câmara coronária; Nível 4 - desgaste total da coroa, até o nível radicular, com separação completa das raízes nos dentes multirradiculados.

Quando referido que um dente foi perdido ante-mortem, isto significou que foi perdido ainda em vida, e que seu alvéolo estava remodelado ou completamente fechado. $\mathrm{O}$ dente foi referido como perdido após a morte, isto é, post-mortem, se seu alvéolo não apresentou nenhum remodelamento (mesmo que o dente tenha sido perdido no momento ou pouco antes da morte, sem tempo para remodelamento do alvéolo).

As classes etárias dentais (c.e.d.) foram determinadas através de método que pode ser utilizado tanto em animais vivos quanto mortos, sendo baseado nas classes etárias dentais ("dental age classes") modificadas por TYNDALE-BISCOE \& MACKENZIE (1976), considerado o método mais acurado para a determinação de idade em Didelphis durante a fase pós-desmame por diversos autores (CORDEIRO \& NICOLAS, 1987; TRIBE, 1990; D'ANDREA et al., 1994; SANTORI et al., 1995). Este método se baseou na presença e no estado funcional dos dentes maxila- res distais aos caninos, a partir do terceiro pré-molar primário e secundário, até o quarto molar, de acordo com a seqüência de erupção e o desgaste das cúspides, consistindo em sete classes etárias dentais: Classe 1 dentes erupcionados: dP3,M1; Classe 2 - dentes erupcionados: dP3,M1,M2; Classe 3 - dentes erupcionados: dP3,M1,M2,M3; Classe 4 - dentes erupcionados: P3,M1,M2,M3; Classe 5 - dentes erupcionados: P3,M1,M2,M3,M4; Classe 6 - dentes erupcionados: P3, M1, M2, M3, M4, com desgaste nos P3 e M1-2; Classe 7 - dentes erupcionados: P3, M1, M2, M3, M4, com desgaste nos P3 e M1-4. As classes etárias podem ser correlacionadas com idades aproximadas, através de comparações de PETRIDES (1949). Assim, o animal da classe etária 1 teria 4 meses; o da 2, entre 5-8 meses; os das 3-4 entre 7-11 meses; os das 5-7 não foram correlacionados com idades aproximadas.

\section{RESULTADOS}

Em relação aos incisivos (Tabela 1), a maior frequiência de dentina terciária foi observada nos dentes mais anteriores, variando de $11,22 \%$ nos I1 e $7,46 \%$ nos i1 até $3,97 \%$ nos I5 e 1,26\% nos i4. Os primeiros pré-molares (Tabela 1), somente apresentaram dentina terciária quando os demais pré-molares e molares estavam muito desgastados, fraturados ou foram perdidos ante-mortem. Isto ocorreu numa freqüência muito baixa $(2,71 \%$ nos $\mathrm{P} 1$ e $1,55 \%$ nos $\mathrm{p} 1)$, tendo sido observado somente em animais adultos, sendo que dos 12 espécimes, 3 eram da c.e.d. (classe etária dental) 6 e 5 da c.e.d. 7. A freqüência de dentina terciária nos terceiros pré-molares primários foi muito baixa (4,39\% nos dP3 e 5,31\% nos dp3), sendo que dos 4 espécimes, dois eram da c.e.d. 2 e dois da c.e.d 3.

Apesar de não haver contato oclusal entre os caninos superiores e inferiores durante a mastigação, mas apenas quando o animal "fricciona" os caninos inferiores contra os inferiores na intenção de produzir som, a freqüência de dentina terciária no terço oclusal foi de $8,81 \%$ nos $\mathrm{C} 1$ e $8,25 \%$ nos c1 (Tabela 1 ). Observou-se com certa freqüência desgaste do esmalte, e algumas vezes também da dentina na face mesial dos caninos superiores e na face distal dos inferiores.

Em relação aos pré-molares e molares, de forma genérica, os dentes que apresentaram maior nível de desgaste, representado pela presença de dentina terciária (Tabela 1), foram os primeiros molares (38,87\% nos M1 e 41,76\% nos m1), seguidos pelos segundos molares $(29,35 \%$ nos M2 e 37,02\% nos m2), os terceiros molares $(20,89 \%$ nos M3 e $29,80 \%$ nos m3) e os terceiros pré-molares $(17,40 \%$ nos P3 e 
Tabela 1 - Dentes superiores e inferiores e alterações encontradas em Didelphis albiventris e D. marsupialis.

\begin{tabular}{|c|c|c|c|c|c|c|c|}
\hline \multirow[t]{2}{*}{ Dentes } & \multirow{2}{*}{$\begin{array}{c}\text { Total de dentes } \\
\text { presentes }\end{array}$} & \multicolumn{2}{|c|}{$\begin{array}{c}\text { Exposição da câmara coronária e/ou } \\
\text { canal radicular }\end{array}$} & \multicolumn{2}{|c|}{$\begin{array}{c}\text { Destruição quase total da } \\
\text { coroa }\end{array}$} & \multicolumn{2}{|c|}{ Dentina terciária } \\
\hline & & Número & $\%$ & Número & $\%$ & Número & $\%$ \\
\hline I1 & 277 & 11 & 3,97 & 19 & 6,85 & 32 & 11,22 \\
\hline $\mathrm{I} 2$ & 287 & 00 & 0,00 & 11 & 3,83 & 32 & 10,77 \\
\hline $\mathrm{I} 3$ & 294 & 01 & 0,34 & 10 & 3,40 & 32 & 10,63 \\
\hline I4 & 295 & 02 & 0,67 & 09 & 3,05 & 20 & 6,57 \\
\hline I5 & 294 & 00 & 0,00 & 20 & 6,80 & 12 & 3,97 \\
\hline $\mathrm{C}$ & 324 & 52 & 16,04 & 04 & 1,23 & 29 & 8,81 \\
\hline $\mathrm{P} 1$ & 324 & 05 & 1,60 & 15 & 4,62 & 09 & 2,71 \\
\hline $\mathrm{P} 2$ & 333 & 07 & 2,10 & 06 & 1,80 & 52 & 15,33 \\
\hline P3 & 241 & 08 & 3,31 & 05 & 2,07 & 43 & 17,40 \\
\hline dP3 & 94 & 01 & 1,06 & 00 & 0,00 & 04 & 4,39 \\
\hline M1 & 333 & 03 & 0,90 & 09 & 2,70 & 131 & 38,87 \\
\hline M2 & 322 & 03 & 0,93 & 09 & 2,79 & 96 & 29,35 \\
\hline M3 & 261 & 00 & 0,00 & 02 & 0,76 & 56 & 20,89 \\
\hline M4 & 217 & 04 & 1,84 & 00 & 0,00 & 24 & 10,48 \\
\hline TOTAL & 3896 & 97 & $\mathrm{xxx}$ & 119 & $\mathrm{XxX}$ & 572 & $\mathrm{xxx}$ \\
\hline i1 & 308 & 06 & 1,94 & 09 & 2,92 & 23 & 7,46 \\
\hline i2 & 314 & 06 & 1,91 & 05 & 1,59 & 18 & 5,73 \\
\hline i3 & 321 & 04 & 1,24 & 07 & 2,18 & 14 & 4,36 \\
\hline i4 & 315 & 01 & 0,31 & 17 & 5,39 & 04 & 1,26 \\
\hline $\mathrm{c}$ & 327 & 12 & 3,66 & 10 & 3,05 & 27 & 8,25 \\
\hline $\mathrm{p} 1$ & 321 & 04 & 1,24 & 36 & 11,21 & 05 & 1,55 \\
\hline p2 & 329 & 06 & 1,82 & 11 & 3,34 & 37 & 11,24 \\
\hline p3 & 235 & 04 & 1,70 & 05 & 2,12 & 34 & 14,46 \\
\hline $\mathrm{dp} 3$ & 94 & 01 & 1,06 & 01 & 1,06 & 05 & 5,31 \\
\hline $\mathrm{m} 1$ & 328 & 07 & 2,13 & 08 & 2,43 & 137 & 41,76 \\
\hline $\mathrm{m} 2$ & 316 & 02 & 0,63 & 07 & 2,21 & 117 & 37,02 \\
\hline $\mathrm{m} 3$ & 255 & 04 & 1,56 & 00 & 0,00 & 76 & 29,80 \\
\hline $\mathrm{m} 4$ & 251 & 04 & 1,59 & 00 & 0,00 & 28 & 11,15 \\
\hline TOTAL & 3714 & 61 & $\mathrm{xxx}$ & 116 & $\mathrm{Xxx}$ & 525 & $\mathrm{xxx}$ \\
\hline
\end{tabular}

$14,46 \%$ nos p3). Somente quando os demais dentes pré-molares e molares estavam muito desgastados (nível 3 ou 4) ou fraturados, os segundos pré-molares $(15,33 \%$ nos $\mathrm{P} 2$ e $11,24 \%$ nos p2) e os quarto molares $(10,48 \%$ nos M4 e 11,15\% nos $\mathrm{m} 4)$ começaram a apresentar desgaste. Apesar desta ter sido a seqüência padrão de desgaste, houve várias exceções, como por exemplo, num dos espécimes, no qual os pré-molares e molares apresentaram dentina terciária, porém não os P3 e M1 direitos.

Em relação à idade dos espécimes, quando se relacionou classe etária dental com a porcentagem de espécimes com dentina terciária, observou-se que nos espécimes da classe etária dental 1 não foi identificado nenhum espécime com dentina terciária nos prémolares e/ou molares e apenas dois espécimes $(6,45 \%)$ na c.e.d. 2. Na classe etária dental 3, 40\% e na classe etária dentária $4,36,36 \%$ dos espécimes apresentaram dentina terciária em um ou mais dentes pré-molares e/ ou molares, consistindo em um aumento importante em relação às duas primeiras. Mais da metade dos es- pécimes na c.e.d. $5(62 \%)$, quase todos os da c.e.d. 6 $(93,75 \%)$ e todos os da c.e.d. $7(100 \%)$ apresentaram dentina terciária nos pré-molares e/ou molares. Apenas dois espécimes da c.e.d. 2 e dois da c.e.d. 3 apresentaram dentina terciária nos terceiros pré-molares primários ou decíduos.

\section{DISCUSSÃO}

Diferentemente da maioria dos mamíferos, observou-se em Didelphis que o desgaste foi mais intenso e rápido que a formação compensatória de dentina secundária e terciária, muitas vezes ocorrendo exposição da câmara coronária em animais ainda jovens, como relataram CROMPTON \& HIIEMÄE (1970). Acredita-se que o desgaste muito intenso, como pôde ser observado nos espécimes pesquisados, principalmente nas cúspides altas e proeminentes dos molares, predispôs à ocorrência de fraturas, cujas superfícies oclusais foram posteriormente desgastadas, aumentando progressivamente a perda de tecido dental. 
A presença de dentina terciária nos incisivos ocorreu, provavelmente, devido ao uso mais intenso destes na apreensão. Como somente o ato de apreender o alimento não desgasta o dente até o nível de formação de dentina terciária, supõe-se que a presença desta deva-se a fraturas, com posterior desgaste, ou vice-versa. Apesar de os primeiros pré-molares não participarem da mastigação, através do contato oclusal com o dente antagônico, apresentaram dentina terciária (baixa freqüiência), e supõe-se que a presença desta deva-se, provavelmente, a fraturas no terço oclusal seguidas de desgaste. Apesar de ser molariforme, devendo executar funções mastigatórias semelhantes às de um molar e, portanto, sendo muito utilizado na mastigação, a freqüência de terceiros pré-molares primários com dentina terciária, em relação aos demais molares, foi muito baixa. Acredita-se que isto se deva à dieta neste período ser menos dura, constituindo-se, principalmente, de invertebrados, plantas e frutas, conforme haviam relatado CORDERO \& NICOLAS (1987); SANTORI et al. (1995), e ao curto período de tempo em que os $\mathrm{dP} 3 / \mathrm{dp} 3$ estão presentes, isto é, dos 4 até 7-11 meses de idade (PETRIDES, 1949).

A presença de dentina terciária no terço oclusal dos caninos ocorreu, provavelmente, devido a fraturas seguidas de desgaste. A presença de desgaste do esmalte e, algumas vezes, também de dentina na face mesial dos caninos superiores e na face distal dos caninos inferiores, deve ser resultado da abrasão durante vocalizações comportamentais, como relatou GARDNER (1982).

De acordo com HIIEMÄE \& CROMPTON (1971), o molar tribosfênico, com suas cúspides altas e pontiagudas, seria ideal para uma dieta onívora. Porém, se os molares tribosfênicos fossem mesmo "ideais" não seria de se esperar níveis tão importantes de atrição em animais que vivem tão pouco tempo (em média dois anos), nos quais nem mesmo a formação de dentina secundária, comum até em espécimes jovens, é rápida o suficiente para impedir a exposição da câmara coronária. Por outro lado, também não seria de se esperar tantas fraturas dentárias.

Não foi possível determinar a razão da diminuição na frequiência de dentina terciária entre as classes etárias dentais 3 e 4, quando o esperado seria que houvesse um acréscimo de espécimes com dentina terciária.

Conforme havia relatado SPOUGE (1977), a mastigação com dentes que não são substituídos, isto é, um reduzido complexo de dentes, resulta em grande perda de tecido dental e, assim sendo, concorda-se com FERIGOLO (1995), o qual afirma que o grande responsável pelo desgaste tão acentuado em Didelphis seria a substituição de um único dente da arcada (terceiro pré-molar primário).

Observa-se nos estádios ontogenéticos mais avançados de Didelphis, um aumento na freqüência de dentina terciária nos pré-molares e molares, ultrapassando a metade dos espécimes na c.e.d. 5 (62\%), atingindo quase todos da c.e.d. $6(93,75 \%)$, e todos da c.e.d. $7(100 \%)$, concordando com SHAFER et al. (1979) os quais afirmam que o desgaste devido à atrição está associado ao processo normal de envelhecimento.

Apesar de não se poder fazer tal afirmação, devido à falta de estudos em animais vivos e no seu meio ambiente, parece razoável supor, ainda de acordo com FERIGOLO (1995), que animais que perderam quase completamente a coroa dos seus molares e que, portanto, não cortariam mais os alimentos, possam ter tido maiores dificuldades para encontrar alimentos, e que a dor durante a mastigação possa ter limitado seu tempo de vida, assim como a atrição normal é um fator etário-limitante para os eqüinos e ruminantes domésticos (DUBIELZIG, 1990). Como a atrição deve-se ao contato entre os dentes e não com alimentos, pois o esmalte é um tecido extremamente rígido, a hipótese mais provável é que os alimentos mais consistentes (ossos, sementes) fraturem as cúspides, que são posteriormente desgastadas.

Acredita-se que uma ótima função mastigatória permite ao animal selecionar uma ampla variedade de itens alimentares requeridos para uma dieta nutricionalmente balanceada, além de propiciar maior resistência a mudanças adversas na disponibilidade de alimentos. No caso dos espécimes com alto nível de desgaste das cúspides dos molares e pré-molares, responsáveis pelo corte e trituração dos alimentos, deve ter havido restrição na sua dieta alimentar, podendo ter contribuído para a sua morte.

Outras inferências foram impossibilitadas por não ter sido realizado estudo dos padrões de microwear (microdesgaste dentário observável em M.E.V.) em nível das superfícies oclusórias, o que poderia inferir padrão de dieta, e pela falta de maiores dados sobre o material, tais como sobre o conteúdo estomacal, o que poderia indicar a relação do tipo de dieta com o grau de desgaste, e também poderia indicar se lesões dentais extensas de algum modo limitam ou até impedem a utilização de alguns itens alimentares.

\section{CONCLUSÕES}

Os molares tribosfênicos de Didelphis não são os "ideais" para o tipo de dieta do animal, ao con- 
trário do que refere a literatura. Existe certo padrão de desgaste nos dentes pré-molares e molares: primeiro, segundo e terceiro molares, terceiro e segundo prémolar, e quarto molar. Apesar desta ser a sequiênciapadrão de desgaste, há várias exceções. As fraturas nas cúspides dos molares favorecem o desgaste, e este as fraturas nas margens da coroa, e o conjunto dos dois leva à rápida perda da coroa do dente. $\mathrm{O}$ grau de desgaste dentário é cronorrelacionável com a idade dos animais.

\section{AGRADECIMENTOS}

À Fundação Zoobotânica do Rio Grande do Sul (FZBRS), pelo uso de suas dependências durante a realização do estudo e pelo empréstimo do material aqui listado; à PUC-RS, pela oportunidade de realização do mestrado; à CAPES, pelo financiamento da pesquisa, por intermédio da bolsa de estudos; à Universidade Federal de Santa Catarina e ao Museu de História Natural Capão da Imbuia, pelo empréstimo do material; à Agência Goiana de Desenvolvimento Rural e Fundiário (AGENCIARURAL-GO), pelo apoio financeiro.

\section{REFERÊNCIAS BIBLIOGRÁFICAS}

CORDERO, G.A.; NICOLAS, R.A. Feeding habits of the opossum (Didelphis marsupialis) in northern Venezuela. Fieldiana Zoology, v.39, p.125-131, 1987.

CROMPTON, A.W.; HIIEMÄE, K. Molar occlusion and mandibular movements during occlusion in the american opossum, Didelphis marsupialis L. Zoological Journal Linnean Society, v.49, p.21-47, 1970.

D'ANDREA, P.S.; CERQUEIRA, R; HINGST, E.D. Age estimation of the gray four eyed opossum, Philander opossum (Didelphimorphia:Didelphidae). Mammalia, v.58, n.2, p.283-291, 1994.

DAVIES, T.G.H; PEDERSEN, P.O. The degree of attrition of the deciduous teeth and first permanent molars of primitive and urbanised greenland natives. British Dental Journal, v.99, n.2, p.35-43, 1955.

DUBIELZIG, R.R. Patologia dental. In: THOMSON, R.G. Patologia veterinária especial. São Paulo : Manole, 1990. Cap.3, p.143-153.

FERIGOLO, J. Relação entre tempo de vida máximo e patologias dentárias em Didelphis (Mammalia, Marsupialia) do Rio Grande do Sul, Brasil. In: JORNADAS ARGENTINAS DE MASTOZOOLOGÍA 10., 1995, La Plata, Argentina. Anais... La Plata : Sociedad Argentina para el Estudio de los Mamíferos, 1995. p.24.

GARDNER, A.L. Virginia opossum - Didelphis virginiana. In: CHAPMAN, J.A.; FELDHAMER, G.A. Wild mammals of North America. Baltimore : Johns Hopkins, 1982. Cap.1, p.3-36.

GIOSO, M.A. Afecções periodontais. Cadernos Técnicos da UFMG, Belo Horizonte, n.20, p.71-80, jun, 1997.

GIOSO, M.A. Odontologia para o clínico de pequenos animais. São Paulo : FMVZ-USP, 2002. 86p.

HIIEMÄE, K.; CROMPTON, A.W. A cinefluorographic study of feeding in the american opossum, Didelphis marsupialis. In: DAHLBERG, A.A. Dental morphology and evolution. Chicago : University of Chicago, 1971. Cap.17. p.299-335.

KAZIMIROFF,T. A report on the dental pathology found in animals that diet in the New York Zoological Park in 1938. Zoologica NY Zool Soc, Nova York, v.24, n.14, p.297-304, 1938.

PAULA-COUTO, C. de. Tratado de paleomastozoologia. Rio de Janeiro : Academia Brasileira de Ciências, 1979. 590p.

PETRIDES, G.A. Sex and age determination in the opossum. Journal of Mammalogy, v.30, n.4, p.364-378, 1949.

ROBINSON, P.T. Oral pathology in mammals at the San Diego Zoo and Wild Animal Park. Proc Am Assoc Zoo Vet, p.96-98, 1979.

SANTORI, R.T.; ASTÚADE MORAES D.; CERQUEIRA, R. Diet composition of Metachirus nudicaudatus and Didelphis aurita (Marsupialia, Didelphoidea) in southeastern Brazil. Mammalia, v.59, n.4, p.511-516, 1995.

SHAFER, W.G.; HINE, M.K.; LEVY, B.M. Patología bucal. Rio de Janeiro : Interamericana, 1979. 728p.

SPOUGE, J.D. Patología bucal. Buenos Aires : Mundi, 1977. $485 \mathrm{p}$.

TRIBE, C.J. Dental age classes in Marmosa incana and other didelphoids. Journal of Mammalogy, v.71, n.4, p.566-569, 1990.

TYNDALE-BISCOE, C.H.; MACKENZIE, R.B. Reproduction in Didelphis marsupialis and D. albiventris in Colombia. Journal of Mammalogy, v.57, n.2, p.249-265, 1976.

WIGGS, R.B.; LOBPRISE, H.B. Veterinary dentistry. Principles and practice. New York : Lippincott- Raven, 1997. 556p. 